\title{
Efeitos da ligadura do ducto pancreático e da secção ductal com livre drenagem de secreções para o peritônio sobre as funções endócrina e exócrina do pâncreas. Estudo clínico e laboratorial em coelhos ${ }^{1}$
}

Endocrine and exocrine consequences of the open and ligated pancreatic duct: clinical and laboratorial findings in rabbits

\section{Lauro Bogodar Kuczynski ${ }^{2}$, César Tadeu Spadella ${ }^{3}$, Carlos Roberto Padovanni ${ }^{4}$}

1. Trabalho realizado no Laboratório de Técnica Cirúrgica e Cirurgia Experimental da Faculdade de Medicina de Botucatu - UNESP; parte integrante na Tese de Doutorado apresentada ao Curso de PósGraduação em Cirurgia "Bases Gerais da Cirurgia e Cirurgia Experimental".

2. Professor Doutor pela Faculdade de Medicina de Botucatu - UNESP e Cirurgião do Aparelho Digestivo do Hospital São Vicente em Curitiba - PR.

3. Professor Adjunto da Disciplina de Gastroenterologia Cirúrgica do Departamento de Cirurgia e Ortopedia da Faculdade de Medicina de Botucatu - UNESP.

4. Professor Titular do Departamento de Estatística do Instituto de Biociências do Campus da UNESP Botucatu.

\section{RESUMO}

As ressecções pancreáticas seguidas de anastomoses acompanham-se de altas taxas de morbidade, que incluem: surtos de pancreatite e, principalmente, fístulas digestivas. Nos transplantes pancreáticos a drenagem da parte exócrina do enxerto para o intestino ou para a bexiga, além das peculiaridades técnicas, também não é isenta de diversas complicações. Visando evitar ou atenuar tais consequiências e simplificar a técnica cirúrgica, têm sido usadas outras abordagens para o tratamento ductal do coto/enxerto pancreático, tais como: drenagem livre de secreções para o peritônio, com o ducto pancreático aberto, ligadura ductal e oclusão do ducto com polímeros sintéticos.

OBJETIVO: O presente estudo visa avaliar clínica e laboratorialmente as funções endócrina e exócrina do pâncreas de coelhos com o ducto aberto e ligado.

MÉTODOS: Foram realizadas 150 operações, divididas em 3 grupos: N manipulação/controle $(n=50), A$ - grupo aberto $(n=50)$, e $\mathrm{L}$ - ligado $(\mathrm{n}=50)$. Os momentos de observação foram pré-operatório, dia 0 (dia da operação) e pós-operatório (observação e sacrifício): 7 dias, 14 dias, 28 dias, 90 dias e 180 dias. Os parâmetros analisados foram: estado geral, atividade, controles do peso corporal, ingestão hídrica, ingestão alimentar e dosagens da amilase sangüínea, glicemia, glicose urinária e insulina plasmática.

RESULTADOS: Todos os grupos tiveram evolução clínica similar, com bom estado geral, ganho ponderal progressivo e valores normais da ingestão hídrica e ingestão alimentar. Exceto uma significativa elevação da amilase sangüínea nas primeiras $24-48 \mathrm{~h}$ para os 2 grupos com interferência ductal, não houve qualquer alteração dos níveis basais da glicemia, glicosúria e insulinemia entre os 3 grupos experimentais, em todos os momentos de observação.

CONCLUSÃO: À exceção da amilase sangüínea, cujos níveis basais foram significativamente elevados no $1^{\underline{0}}$ e $2^{\underline{0}}$ dias de pós-operatório, as duas modalidades técnicas de abordagem da secreção exócrina do pâncreas, utilizadas em coelhos, não determinaram 
quaisquer alterações clínicas, bem como dos níveis basais de glicose sanguínea, glicose urinária e insulina plasmática, durante 6 meses de seguimento.

Descritores: Pâncreas. Ligadura ductal. Drenagem ductal livre. Peritônio.

\begin{abstract}
After pancreatic ressections due to chronic pancreatitis the remnant pancreas can lead to new outbreaks of pancreatitis in variable degrees of severity. After the resections by tumors or pancreatitis, the most common complications are the pancreatic fistulas with their resultant morbidity and mortality. In the pancreas transplantation the bowel or bladder drainage of the exocrine part of the graft, beyond the technical peculiarities of the execution, is not exempt of several complications. In order to avoid or reduce such consequences and trying to simplify the surgical technique, there have been used other approaches for the treatment of the pancreatic stump/graft: free drainage of the secretions with the duct left open, ductal ligature and duct occlusion with polymers.

PURPOSE: The proposal of our investigation was to study the clinical and laboratorial effects of the ductal ligature and the ductal left open in the endocrine and exocrine compounds of the rabbit pancreas.

METHODS: One hundred and fifty operations were performed, divided in 3 groups: laparotomy/control $(n=50)$, opened group $(n=50)$ and ligated $(n=50)$. The moments of observation were pre-operative, day 0 (operation's day) and post-operative (observation and sacrifice): 7 days, 14 days, 28 days, 90 days and 180 days. The parameters analysed were: general clinic state, activity and controls of body weight, water intake, food intake and measurements of blood and urinary glucose, serum-amylase and plasma insulin levels. RESULTS: All the groups had a similar clinical evolution: progressive body weight gain, water and food intake, as well as in good health conditions.

CONCLUSIONS: Except for a significant serum-amylase elevation in the first 24-48 hours for the two ductal interference groups, there were no other blood glucose and insulin and urine-glucose variations in the 3 groups, in all observation moments.
\end{abstract}

Key words: Pancreas. Pancreas ductal ligature. Duct left open. Peritoneum.

\title{
Introdução
}

Apesar dos avanços científicos e do aperfeiçoamento da técnica operatória, o tratamento cirúrgico das patologias do pâncreas, particularmente as ressecções e as derivações pancreáticas, ainda acompanham-se de altas taxas de morbi-mortalidade ${ }^{1,2,3,4,5}$.

A mortalidade associada às ressecções pancreáticas, seguidas ou não de anastomoses, atinge índices que chegam até $20 \%{ }^{6,7,8}$, sendo que a fístula pancreática adiciona morbidade que oscila entre 5 e $15 \%$ nas pancreatectomias ${ }^{9,5}$. 
As derivações pancreatojejunais, sem ressecção pancreática, também acompanham-se de elevados índices de fístulas pancreáticas que variam entre 8 e $17 \%{ }^{10,8}$, com mortalidade tardia de até $29.2 \%^{11,12}$.

A morbidade relacionada aos efeitos danosos da secreção exócrina do pâncreas também está presente nas derivações entéricas ${ }^{13}$ e nas derivações para o trato urinário ${ }^{14}$ de pacientes submetidos ao transplante pancreatoduodenal heterotópico, com finalidade terapêutica do diabetes mellitus tipo 1.

No intuito de evitar tais conseqüências têm sido usadas outras formas de abordagem cirúrgica da parte exócrina do pâncreas, tais como: ligadura do ducto pancreático principal, oclusões ductais com polímero sintético e livre drenagem de secreções pancreáticas para a cavidade peritoneal. Todavia, o efeito destas modalidades de tratamento do ducto pancreático ainda é bastante discutível, principalmente em relação aos possíveis danos causados na função endócrina e/ou exócrina do pâncreas remanescente e de enxertos pancreáticos.

Assim, no intuito de esclarecer as dúvidas reportadas aqui, delineamos o presente trabalho de pesquisa com o objetivo de avaliar, a longo prazo, se a ligadura e a livre drenagem de secreções pancreáticas para o peritônio interferem com a evolução clínica e laboratorial de coelhos.

\section{Métodos}

Animais utilizados - No presente trabalho foram utilizados 150 coelhos da linhagem Norfolk, adultos, machos, sadios ao exame clínico, pesando, em média, entre 1700 e 2000g, com aproximadamente 2 meses de idade, fornecidos pelo Biotério Central do Campus da UNESP de Botucatu.

Grupos experimentais - Os animais foram distribuídos por sorteio em três grupos experimentais, assim designados: Grupo N - constituído de 50 animais, submetidos à cirurgia simulada; Grupo A - constituído de 50 animais, submetidos à secção distal do ducto pancreático, com livre drenagem da secreção pancreática para o peritônio; Grupo L constituído de 50 animais, submetidos à ligadura distal do ducto pancreático.

Delineamento experimental - Os animais foram alojados no Laboratório de Cirurgia Experimental, aos pares, em gaiolas galvanizadas, de 50 × 50 x $50 \mathrm{~cm}$ de tamanho, com oferecimento de ração Nutri-coelho (Agribrands do Brasil Ltda - Paulínia, SP) e água "ad 
libitum". Nestas condições, os mesmos permaneceram por um período de adaptação de 7 dias, período em que foram observados e examinados clinicamente, sendo também tratados profilaticamente de verminoses com Mebendazole $(25 \mathrm{mg} / \mathrm{kg}$ de peso/dia), adicionado na água de beber, durante 3 dias. As condições de temperatura, iluminação (noite/dia) e exaustão do ambiente foram controladas artificialmente durante todo o experimento.

Terminado o período de adaptação, os animais foram identificados e pesados, tendo sido submetidos ao primeiro sorteio para constituição dos três grupos experimentais (manipulado, aberto e ligado), com 50 coelhos cada um.

Dentro dos respectivos grupos experimentais, os animais foram submetidos ao procedimento cirúrgico preconizado, no dia seguinte ao sorteio; sendo após a cirurgia, novamente sorteados para a definição dos períodos de sacrifício, respectivamente após 7 , $14,28,90$ e 180 dias de seguimento.

Parâmetros analisados - Clínicos: observação da atividade, aspecto clínico geral, aspecto da pelagem, apetite e medidas da evolução da curva ponderal, da ingestão hídrica e da ingestão alimentar. Também foi observado o aspecto das incisões abdominais (infecção, deiscência, herniações). Laboratoriais: foram realizadas dosagens da amilase sangüínea, glicose sangüínea de jejum, glicose urinária de 24h e níveis basais de insulina plasmática.

Momentos de avaliação e sacrifício - Todos os parâmetros clínicos e laboratoriais, à exceção da dosagem da amilase sanguiínea, que também foi dosada 24 e $48 \mathrm{~h}$ após a cirurgia, foram avaliados nos seguintes momentos do experimento: $\mathrm{M}_{0}$ - momento inicial, com parâmetros obtidos no dia da cirurgia preconizada; $\mathrm{M}_{1}$ - no sacrifício, após 7 dias de pós-operatório; $\mathrm{M}_{2}$ - no sacrifício, após 14 dias de pós-operatório; $\mathrm{M}_{3}$ - no sacrifício, após 28 dias de pós-operatório; $M_{4}$ - no sacrifício, após 90 dias de pós-operatório e $\mathrm{M}_{5}$ - no sacrifício, após 180 dias de pós-operatório. Em cada um destes cinco momentos, 10 coelhos foram sacrificados por subgrupo, em cada grupo experimental, totalizando 50 animais/grupo experimental (10x5 momentos).

Metodologia utilizada para a obtenção de parâmetros clínicos e laboratoriais - Cada animal do experimento foi avaliado seis vezes, como definido acima. Nestas oportunidades, os animais seguiram uma rotina de procedimentos para a coleta dos dados, que obedeceu a seguinte metodologia: a) colocação dos animais em gaiolas individuais, $24 \mathrm{~h}$ antes do dia da cirurgia; b) fornecimento de volume conhecido de água durante todo o 
período de $24 \mathrm{~h}$; c) fornecimento de quantidade conhecida de ração durante o período noturno de $12 \mathrm{~h}$, sendo que após este, foi retirado toda a alimentação por um período de $12 \mathrm{~h}$ (jejum de 12 horas); d) determinação ao final do procedimento: do peso animal, em gramas; do volume de água ingerido em $24 \mathrm{~h}$, em mililitros; da quantidade de alimento consumido em 12h, em gramas; sendo a diurese de $24 \mathrm{~h}$ não quantificada por impossibilidade técnica e indisponibilidade de material; e) coleta de uma amostra de $10 \mathrm{ml}$ da urina obtida em $24 \mathrm{~h}$, para a dosagem da glicose urinária, em miligramas por decilitro; f) coleta, por punção venosa das orelhas $\left(\mathrm{M}_{0}, 1^{\underline{0}}\right.$ e $2^{\underline{0}}$ P.O.) ou punção cardíaca $\left(\mathrm{M}_{1}, \mathrm{M}_{2}\right.$, $\mathrm{M}_{3}, \mathrm{M}_{4}, \mathrm{M}_{5}$ ), de amostras de sangue para a dosagem de glicose plasmática, em miligramas por decilitro, após um jejum de $12 \mathrm{~h}$, bem como de amilase sangüínea, em Unidades Amilolíticas por decilitro e insulina plasmática, em microunidades por mililitro.

Técnicas utilizadas - Pré-operatório: todos os coelhos foram mantidos em jejum alimentar de 12 horas, sendo liberada apenas a água. No pré-operatório imediato, todos os animais receberam antibioticoterapia profilática, utilizando a cefazolina - Kefazol (Eli Lilly do Brasil Ltda. - São Paulo, SP) na dose única de $50 \mathrm{mg} / \mathrm{kg}$ de peso, administrada por via intramuscular. Anestesia: para a realização dos procedimentos cirúrgicos e o sacrifício dos animais, foi utilizada a anestesia endovenosa com pentobarbital sódico - Hipnol 3\% (Cristália - Produtos Químicos e Farmacêuticos Ltda. - Itapira, SP), na dose de $30 \mathrm{mg} / \mathrm{kg}$ de peso corporal, utilizando-se uma das veias auriculares. Cirurgia: independentemente do tipo de operação, todos os animais foram submetidos à ampla tricotomia, anti-sepsia com álcool iodado a 2\%; paramentação cirúrgica, campos e instrumentais estéreis. A seguir, foi realizada laparotomia mediana supra e infra-umbilical de aproximadamente $8 \mathrm{~cm}$ de extensão, com liberação e manipulação do duodeno, em cujo mesentério vascular se aloja o pâncreas (Figura 1A), seguido de exposição do ducto pancreático (Figura 1B). Esta foi a tática para o grupo manipulado/ normal. No grupo aberto, para se evitar a obliteração do ducto pelas aderências que se seguem à secção, o mesmo foi cateterizado com uma sonda de polivinil P.E. no 100 (Hemo Técnico - Artefatos de Plástico Ltda. - São Paulo, SP) de $3 \mathrm{~cm}$ de extensão, sendo $2 \mathrm{~cm}$ para dentro do ducto e $1 \mathrm{~cm}$ para fora, fixado com seda 4-0 (Figura 2A). Para o grupo ligado usou-se secção entre duas ligaduras com seda 4-0, rente ao duodeno (Figura 2B). O peritônio e o plano músculo-aponeurótico foram fechados em conjunto, com sutura contínua de Vicryl 3-0 e o sub-cutâneo mais a pele com sutura contínua de Mononylon 4-0 (Ethicon - Divisão de Johnson \& Johnson Produtos 
Profissionais Ltda. - São José dos Campos, SP). Pós - Operatório: Após os procedimentos cirúrgicos, todos os animais operados foram mantidos em ambiente aquecido com aquecedor elétrico, durante todo o período de recuperação anestésica. Após acordarem, os coelhos foram conduzidos às suas respectivas gaiolas, com fornecimento de água "ad libitum". A dieta só foi reiniciada após o $1^{\underline{0}}$ dia de pós-operatório, sendo iniciado o seguimento clínico e laboratorial, conforme definido nos itens anteriores.

Métodos utilizados nas dosagens laboratoriais - As dosagens de amilase sérica foram realizadas no Laboratório Clínico da Faculdade de Medicina de Botucatu - UNESP, e no Laboratório Dr. Sugisawa, de Curitiba - PR, empregando-se o método por hidrólise enzimática (MOURA, 1982), através de leituras espectrofotométricas realizadas a 640nm. As dosagens de glicose sangüínea e urinária: foram executadas no Laboratório de Pesquisa Experimental do Departamento de Pediatria da Faculdade de Medicina de Botucatu UNESP, empregando-se o método por dosagem enzimática Glicose-E (CELM Cia. Equipadora de Laboratórios Modernos - Barueri, SP). Os níveis basais de insulina plasmática foram dosados por radioimunoensaio, pelo método em fase sólida COAT-ACOAT INSULIN (DPC - Diagnostics Products Corporation - Los Angeles, USA), realizado no Laboratório de Radioimunoensaio da Disciplina de Endocrinologia da Faculdade de Medicina de Botucatu - UNESP.

Sacrifício dos animais - Em todos os grupos experimentais, os animais foram sacrificados, após anestesia, por meio de hemorragia aguda, conseqüente à punção cardíaca para a coleta de sangue destinado à realização dos exames laboratoriais, e posterior secção da veia porta, e da veia cava, acima da desembocadura das veias hepáticas.

Análise estatística - O estudo das variáveis peso, ingestão hídrica, ingestão alimentar, glicemia de jejum e glicose urinária, nos três grupos experimentais, e nos cinco momentos de sacrifício, foi realizado através da Análise de Variância paramétrica, quando os dados mostraram aderência à distribuição normal de probabilidade, e não paramétrica quando este requisito não estava presente. Em ambas as situações, o teste foi complementado com o procedimento de comparações múltiplas para indicar as significâncias $(\mathrm{P}<0,05)$ entre grupos, fixado momento, e momentos, dentro do grupo; Para a indicação dos resultados do teste de comparações múltiplas foram utilizadas letras minúsculas na comparação dos momentos dentro do grupo, e maiúsculas para comparação entre grupos, fixado o 
momento. A interpretação das letras pode ser feita da seguinte maneira: 1. Duas médias ou medianas seguidas de pelo menos uma mesma letra minúscula, não diferem entre si, no nível de 5\% de significância. Isto é, os momentos de sacrifício respondem igualmente para a variável em comparação dentro do grupo considerado; 2. Duas médias ou medianas seguidas de pelo menos uma mesma letra maiúscula, não diferem entre si, no nível de 5\% de significância. Isto é, os grupos correspondentes às médias (ou medianas), respondem para a variável em observação com a mesma intensidade, dentro do momento fixado.
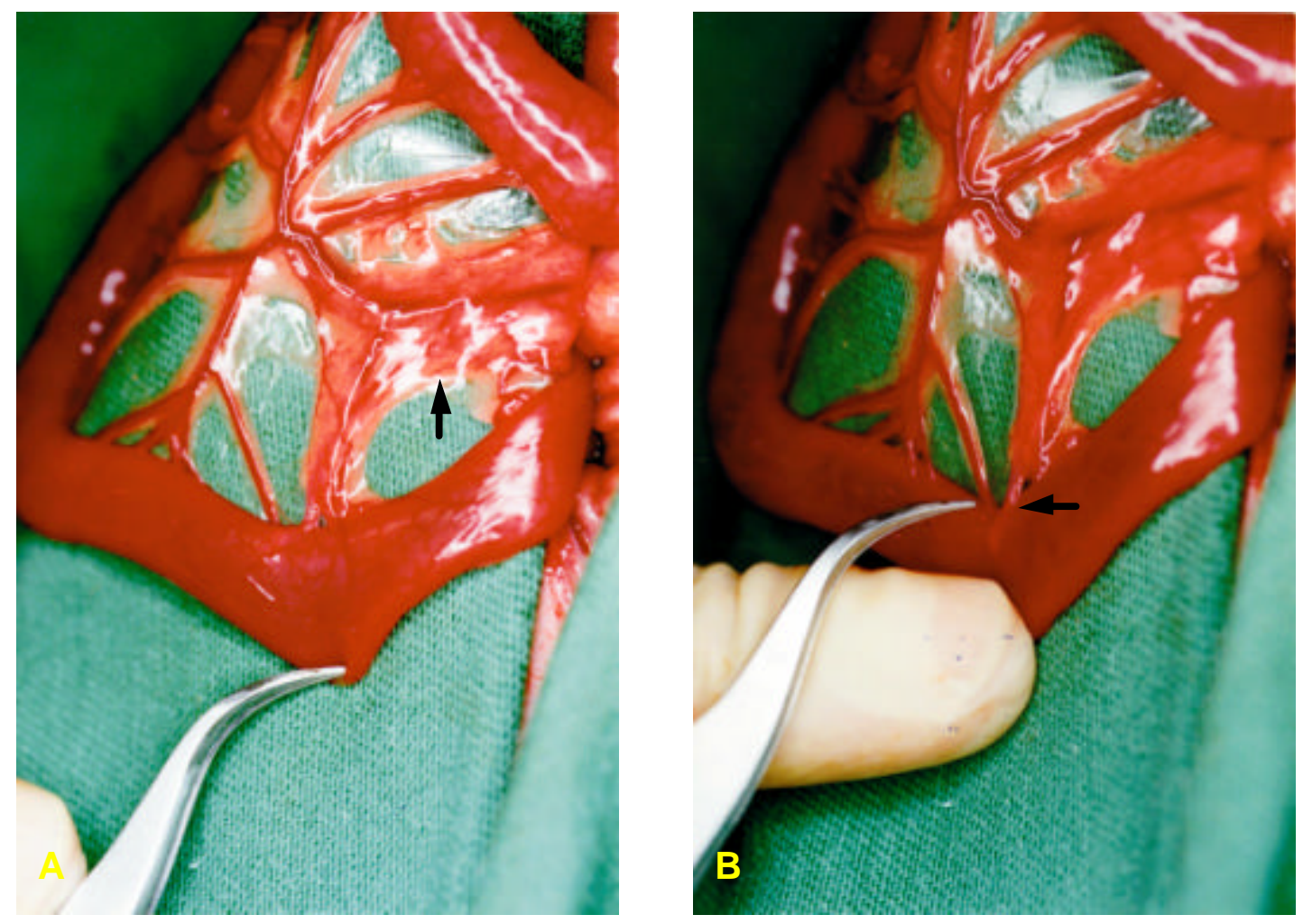

FIGURA 1 - Identificação do pâncreas no mesentério duodenal (A) e da desembocadura do ducto pancreático no duodeno (B). Vide setas. 

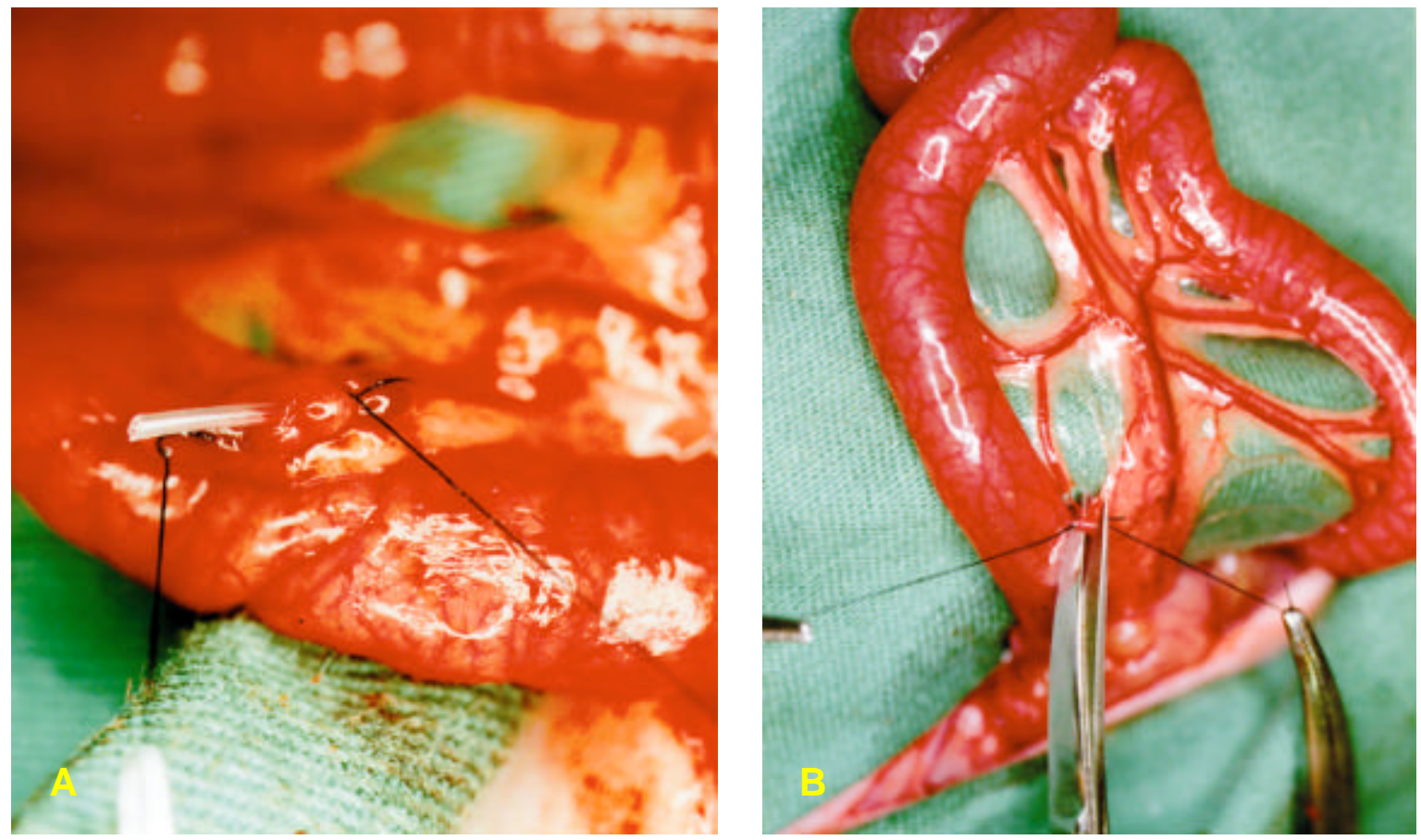

FIGURA 2 - Detalhes do cateterismo do ducto pancreático e da fixação da sonda no interior do ducto. Observe a extremidade distal do cateter deixada $1 \mathrm{~cm}$ para fora do ducto (A) e detalhe da secção distal do ducto pancreático entre duas ligaduras (B).

\section{Resultados}

Quanto à anestesia - A anestesia endovenosa com pentobarbital sódico, na dose de $30 \mathrm{mg} / \mathrm{kg}$ de peso corporal, proporcionou excelentes resultados na realização dos procedimentos cirúrgicos. A indução anestésica foi rápida, com manutenção de plano anestésico satisfatório e suficiente para a execução completa dos tempos cirúrgicos, sem a necessidade, na maioria das vezes, de complementação da dose inicial de anestésico. Quando este fato ocorreu, foi necessário, em média, $1 / 3$ da dose total preconizada.

A anestesia com pentobarbital sódico também proporcionou satisfatória recuperação anestésica, após um tempo médio de 6 horas do término do procedimento cirúrgico. O índice de mortalidade com esse tipo de anestesia foi de 2,6\% (4/150), vinculado principalmente à susceptibilidade do animal e/ou excesso de dose anestésica. Quando o óbito ocorreu, os animais foram repostos.

Peculiaridades na obtenção de um modelo experimental de drenagem livre de secreções pancreáticas para o peritônio do coelho - A secção isolada do ducto pancreático parecianos, inicialmente, o mecanismo óbvio para permitir a drenagem livre de secreções pancreáticas para o peritônio. No entanto, este procedimento mostrou-se ineficaz em produzir os resultados por nós esperados, visto que, após a primeira semana do 
procedimento cirúrgico, ocorria a formação de aderências e fibrose nas adjacências do ducto aberto, as quais determinavam a sua total obliteração. Assim, o ducto que era para ficar aberto, comportava-se como um ducto ligado ou obliterado.

Tentando solucionar este problema optou-se por deixar uma cânula no interior do ducto, com a sua extremidade distal drenando livre na cavidade peritoneal. Esta técnica, embora mais eficaz que a secção isolada, pois permitiu a drenagem livre de secreções pancreáticas por um período maior, também comportou-se, ao cabo de 14-28 dias, como uma técnica de ducto fechado. Neste caso, similarmente à secção isolada, também observamos, na maioria dos animais, a formação de um bloqueio ao redor da cânula, o qual determinava dilatação ductal a montante, impedindo o livre fluxo de secreções para o peritônio. Em nosso experimento, dos 50 animais do grupo aberto, submetidos à técnica de tubagem ductal, apenas 6 animais (12\%) comportaram-se como portadores de técnica com drenagem verdadeiramente livre para a camada peritoneal. Desses, 3 animais foram sacrificados com 28 dias de seguimento, 2 com 3 meses e apenas um animal, com 6 meses de estudo.

Estudos clínicos - Não houve modificação alguma no aspecto geral, pelagem, compleição e vivacidade dos animais no 3 grupos estudados, qualquer que fosse o procedimento. Abscesso de parede foi encontrado em 2 animais do grupo aberto (4\%) e em 2 do grupo ligado (4\%), o que foi facilmente sanado com drenagem e limpeza. A evolução ponderal progressiva ocorreu sem diferenças estatisticamente significativas entre os três grupos (Tabela 1).

Achados clínicos que pudessem sugerir comprometimento exócrino e endócrino do pâncreas, tais como: diarréia, emagrecimento, fezes oleosas, polifagia e polidipsia, não foram observadas quer no grupo normal/manipulado $(\mathrm{N})$ quer nos grupos aberto (A) e ligado (L). Os valores da ingestão hídrica e da ingestão alimentar não apresentaram diferenças estatísticas significativas entre os grupos (Tabelas 2 e 3 ). 
TABELA 1 - Médias, desvios-padrão e resultados do teste estatístico da comparação do peso, em gramas, nos três grupos experimentais e nos momentos de sacrifício.

\section{PESO}

\begin{tabular}{c|c|c|c|c|c|c}
\hline \multicolumn{7}{c}{ Períodos de sacrifício (dias) } \\
\hline \multirow{2}{*}{ Grupos } & $\begin{array}{c}\text { Início } \\
\left(\mathbf{M}_{\mathbf{0}}\right)\end{array}$ & $\mathbf{7}$ & $\mathbf{1 4}$ & $\mathbf{2 8}$ & $\mathbf{9 0}$ & $\mathbf{1 8 0}$ \\
& $\left(\mathbf{M}_{\mathbf{1}}\right)$ & $\left(\mathbf{M}_{\mathbf{2}}\right)$ & $\left(\mathbf{M}_{\mathbf{3}}\right)$ & $\left(\mathbf{M}_{\mathbf{4}}\right)$ & $\left(\mathbf{M}_{\mathbf{5}}\right)$ \\
\hline \multirow{2}{*}{$\mathbf{N}$} & $1858,6 \pm 137,7 \mathrm{a}$ & $1953,7 \pm 163,5 \mathrm{a}$ & $2117,9 \pm 209,8 \mathrm{a}$ & $2520,3 \pm 174,7 \mathrm{~b}$ & $3221,6 \pm 317,3 \mathrm{c}$ & $3375,6 \pm 298,1 \mathrm{c}$ \\
& $\mathrm{A}$ & $\mathrm{A}$ & $\mathrm{A}$ & $\mathrm{A}$ & $\mathrm{A}$ & $\mathrm{A}$ \\
\hline \multirow{2}{*}{$\mathbf{A}$} & $1945,9 \pm 200,7 \mathrm{a}$ & $1994,1 \pm 215,4 \mathrm{a}$ & $2180,4 \pm 174,6 \mathrm{a}$ & $2485,9 \pm 276,2 \mathrm{~b}$ & $3264,2 \pm 303,5 \mathrm{c}$ & $3472,0 \pm 259,7 \mathrm{c}$ \\
\hline & $\mathrm{A}$ & $\mathrm{A}$ & $\mathrm{A}$ & $\mathrm{A}$ & $\mathrm{A}$ & $\mathrm{A}$ \\
\hline $\mathbf{L}$ & $1903,6 \pm 187,9 \mathrm{a}$ & $1956,4 \pm 210,9 \mathrm{a}$ & $2175,9 \pm 195,4 \mathrm{a}$ & $2464,5 \pm 235,1 \mathrm{~b}$ & $3218,4 \pm 342,3 \mathrm{c}$ & $3475,0 \pm 329,3 \mathrm{c}$ \\
\hline
\end{tabular}

TABELA 2 - Médias, desvios-padrão e resultados do teste estatístico da comparação da ingestão hídrica, em mililitros/24h, nos três grupos experimentais e nos momentos de sacrifício.

\section{INGESTÃO HÍDRICA}

\begin{tabular}{c|c|c|c|c|c|c}
\hline \multicolumn{7}{c}{ Períodos de sacrifício (dias) } \\
\hline \multirow{2}{*}{ Grupos } & Início & $\mathbf{7}$ & $\mathbf{1 4}$ & $\mathbf{2 8}$ & $\mathbf{9 0}$ & $\mathbf{1 8 0}$ \\
& $\left(\mathbf{M}_{\mathbf{0}}\right)$ & $\left(\mathbf{M}_{\mathbf{1}}\right)$ & $\left(\mathbf{M}_{\mathbf{2}}\right)$ & $\left(\mathbf{M}_{\mathbf{3}}\right)$ & $\left(\mathbf{M}_{\mathbf{4}}\right)$ & $\left(\mathbf{M}_{\mathbf{5}}\right)$ \\
\hline \multirow{2}{*}{$\mathbf{N}$} & $327,8 \pm 109,3 \mathrm{a}$ & $312,0 \pm 99,8 \mathrm{a}$ & $357,8 \pm 85,6 \mathrm{a}$ & $342,6 \pm 96,5 \mathrm{a}$ & $331,3 \pm 99,9 \mathrm{a}$ & $316,7 \pm 77,1 \mathrm{a}$ \\
& $\mathrm{A}$ & $\mathrm{A}$ & $\mathrm{A}$ & $\mathrm{A}$ & $\mathrm{A}$ & $\mathrm{A}$ \\
\hline \multirow{2}{*}{$\mathbf{A}$} & $320,6 \pm 80,8 \mathrm{a}$ & $302,2 \pm 78,2 \mathrm{a}$ & $313,6 \pm 72,5 \mathrm{a}$ & $373,4 \pm 97,4 \mathrm{a}$ & $321,0 \pm 91,9 \mathrm{a}$ & $345,0 \pm 107,3 \mathrm{a}$ \\
\hline & $\mathrm{A}$ & $\mathrm{A}$ & $\mathrm{A}$ & $\mathrm{A}$ & $\mathrm{A}$ & $\mathrm{A}$ \\
\hline
\end{tabular}

TABELA 3 - Medianas, semi-amplitudes interquartílicas e valores mínimos e máximos da ingestão alimentar, em gramas/12h, segundo grupos e momentos de sacrifício e os resultados dos testes estatísticos. 


\section{INGESTÃO ALIMENTAR}

\begin{tabular}{c|c|c|c|c|c|c}
\hline \multicolumn{7}{c}{ Períodos de sacrifício (dias) } \\
\hline \multirow{2}{*}{ Grupos } & $\begin{array}{c}\text { Início } \\
\left(\mathbf{M}_{\mathbf{0}}\right)\end{array}$ & $\mathbf{7}$ & $\mathbf{1 4}$ & $\mathbf{2 8}$ & $\mathbf{9 0}$ & $\mathbf{1 8 0}$ \\
& $\left(\mathbf{M}_{\mathbf{1}}\right)$ & $\left(\mathbf{M}_{\mathbf{2}}\right)$ & $\left(\mathbf{M}_{\mathbf{3}}\right)$ & $\left(\mathbf{M}_{\mathbf{4}}\right)$ & $\left(\mathbf{M}_{\mathbf{5}}\right)$ \\
\hline \multirow{4}{*}{$\mathbf{N}$} & $104,1 \pm 44,2 \mathrm{a}$ & $110,7 \pm 38,6 \mathrm{a}$ & $130,3 \pm 25,2 \mathrm{a}$ & $133,5 \pm 34,1 \mathrm{a}$ & $127,4 \pm 43,8 \mathrm{a}$ & $122,2 \pm 31,1 \mathrm{a}$ \\
& $(72,7-135,5)$ & $(83,3-138,1)$ & $(112,3-148,2)$ & $(109,3-157,7)$ & $(96,2-158,5)$ & $(100,1-144,3)$ \\
& $\mathrm{A}$ & $\mathrm{A}$ & $\mathrm{A}$ & $\mathrm{A}$ & $\mathrm{A}$ & $\mathrm{A}$ \\
\hline \multirow{2}{*}{$\mathbf{A}$} & $105,7 \pm 31,9 \mathrm{a}$ & $124,5 \pm 115,2 \mathrm{a}$ & $121,5 \pm 24,2 \mathrm{a}$ & $169,5 \pm 102,3 \mathrm{a}$ & $148,2 \pm 39,7 \mathrm{a}$ & $108,5 \pm 47,0 \mathrm{a}$ \\
& $(83,0-128,4)$ & $(42,7-206,3)$ & $(104,3-138,7)$ & $(96,8-242,1)$ & $(119,9-176,4)$ & $(75,1-141,9)$ \\
& $\mathrm{A}$ & $\mathrm{A}$ & $\mathrm{A}$ & $\mathrm{A}$ & $\mathrm{A}$ & $\mathrm{A}$ \\
\hline \multirow{4}{*}{$\mathbf{L}$} & $109,8 \pm 36,7 \mathrm{a}$ & $105,0 \pm 30,3 \mathrm{a}$ & $126,4 \pm 21,9 \mathrm{a}$ & $137,2 \pm 32,4 \mathrm{a}$ & $135,7 \pm 26,8 \mathrm{a}$ & $115,0 \pm 25,9 \mathrm{a}$ \\
& $(83,7-135,9)$ & $(83,5-126,6)$ & $(110,9-142,0)$ & $(114,3-160,2)$ & $(116,6-154,7)$ & $(96,6-133,4)$ \\
& $\mathrm{A}$ & $\mathrm{A}$ & $\mathrm{A}$ & $\mathrm{A}$ & $\mathrm{A}$ & $\mathrm{A}$ \\
\hline
\end{tabular}

\section{Estudos laboratoriais}

Amilase - A atividade da amilase em coelhos normais manipulados foi um dos parâmetros de referência utilizados para medir os efeitos dos diferentes tratamentos do ducto pancreático sobre o componente exócrino do pâncreas.

Em coelhos normais manipulados, os valores desta enzima oscilaram, em média, entre 203,7 e 229,1 Unidades Amilolíticas por decilitro (UA/dL), mantendo-se, praticamente, sem muitas variações durante todo o seguimento dos animais. Este dado indicou que nem a anestesia, nem a laparotomia com manipulação de vísceras, exerceram influências significativas sobre os níveis desta enzima em coelhos, tomando-se por base os valores da amilase sangüínea obtidos em animais normais, não manipulados.

Por outro lado, a amilasemia nos animais do grupo aberto foi significativamente elevada quando comparada aos valores observados em coelhos normais manipulados $(\mathrm{P}<0,05)$, no $1^{\underline{0}}$ e $2^{\underline{0}}$ dia após a secção do ducto pancreático, atingindo, respectivamente, valores médios de 3044,6 e 1265,9 UA/dL. A partir destes períodos, os valores da amilase diminuíram progressivamente até o $7^{\circ} \mathrm{PO}$, para manterem-se dentro dos níveis de normalidade até o final do experimento (Tabela 4).

Similarmente ao grupo aberto, os valores da amilase sanguiínea no ducto ligado foram significativamente elevados $(\mathrm{P}<0,05)$ no $1^{\mathrm{O}}$ e $2^{2}$ dia após a ligadura ductal, quando comparados aos valores encontrados em coelhos normais manipulados. Da mesma forma, 
estes valores, inicialmente elevados, foram progressivamente diminuindo até o $7^{\circ}$ PO, para alcançarem valores compatíveis com a normalidade, até o final do experimento.

Glicemia, glicosúria e insulina plasmática - As dosagens da glicose sanguiínea e urinária, bem como dos níveis séricos basais de insulina, foram os parâmetros laboratoriais utilizados como referência da atividade endócrina do pâncreas e do seu controle sobre o metabolismo dos carboidratos. Em coelhos normais manipulados, a glicose sangüínea e urinária se mantiveram dentro do padrão de normalidade considerado ideal para coelhos sadios de mesma linhagem e idade de seguimento, respectivamente, em torno de 126-145 $\mathrm{mg} / \mathrm{dL}$ e abaixo de $20 \mathrm{mg} / \mathrm{dL}$. O mesmo ocorreu com os níveis séricos basais de insulina, cujos valores normais oscilaram, em média, entre 9,3 - 11,8 $\mu \mathrm{U} / \mathrm{ml}$, durante todo o experimento (Tabelas 5, 6 e 7).

A secção do ducto pancreático, com drenagem livre de secreções diretamente para o peritônio, também não alterou significativamente os valores da glicose sanguínea e urinária, e os níveis basais de insulina plasmática, durante todo o seguimento, em todos os animais operados. Esta constatação foi verificada tanto em animais cujos ductos permaneceram abertos, por períodos variáveis de tempo (6 animais), como naqueles cujos ductos obliteraram-se por fibrose (44 animais). Os dados destes parâmetros estão apresentados nas tabelas (5, 6 e 7), juntamente com os achados dos demais grupos experimentais.

No grupo ligado os níveis de glicose no sangue e na urina também não se alteraram após o procedimento, em todos os momentos de avaliação, durante todo o seguimento. $\mathrm{O}$ mesmo ocorreu com a insulina plasmática, cujos valores não diferiram dos observados em coelhos normais manipulados, até o final do experimento (Tabelas 5, 6 e 7).

TABELA 4 - Médias, desvios-padrão e resultados do teste estatístico da comparação da amilase sérica, em Unidades Amilolíticas por decilitro, nos grupos experimentais e momentos de sacrifício. 
AMILASE

\begin{tabular}{|c|c|c|c|}
\hline \multirow{2}{*}{$\begin{array}{c}\text { Sacrifício } \\
\text { (dias) }\end{array}$} & \multicolumn{3}{|c|}{ Grupos experimentais } \\
\hline & $\mathbf{N}$ & $\mathbf{A}$ & $\mathbf{L}$ \\
\hline Início (M0) & $\begin{array}{c}209,0 \pm 32,3 a \\
\text { A }\end{array}$ & $\begin{array}{c}220,5 \pm \\
\mathrm{A}\end{array}$ & $\begin{array}{c}209,6 \pm \\
\mathrm{A}\end{array}$ \\
\hline 1 (MA) & $\begin{array}{c}203,7 \pm 39,7 \mathrm{a} \\
\mathrm{A}\end{array}$ & $\begin{array}{c}3044,6 \pm 2417,7 \mathrm{~b} \\
\mathrm{~B}\end{array}$ & $\begin{array}{c}2768,9 \pm 1354,3 b \\
\text { B }\end{array}$ \\
\hline $2(\mathrm{MB})$ & $\begin{array}{c}216,4 \pm 34,0 \mathrm{a} \\
\mathrm{A}\end{array}$ & $\begin{array}{c}1265,9 \pm 558,3 \mathrm{c} \\
\mathrm{B}\end{array}$ & $\begin{array}{c}1107,3 \pm 473,9 c \\
B\end{array}$ \\
\hline 7 (M1) & $\begin{array}{c}220,1 \pm 40,8 \mathrm{a} \\
\mathrm{A} \\
\end{array}$ & $\begin{array}{c}584,9 \pm \\
\text { B } \\
\end{array}$ & $\begin{array}{c}361,8 \pm \\
A \\
A\end{array}$ \\
\hline 14 (M2) & $\begin{array}{c}220,1 \pm 42,9 a \\
\mathrm{~A}\end{array}$ & $\begin{array}{c}180,6 \pm \\
\mathrm{A}\end{array}$ & $\begin{array}{c}190,0 \pm \\
\mathrm{A}\end{array}$ \\
\hline 28 (M3) & $\begin{array}{c}229,1 \pm 40,6 a \\
A\end{array}$ & $\begin{array}{c}183,0 \pm{ }_{A} \\
43,1 a\end{array}$ & $\begin{array}{c}171,5 \pm{ }_{A} \\
25,4 a\end{array}$ \\
\hline 90 (M4) & $\begin{array}{c}220,9 \pm 40,3 a \\
\text { A }\end{array}$ & $\begin{array}{c}171,7 \pm \\
\mathrm{A}\end{array}$ & $\begin{array}{c}173,4 \pm \\
\mathrm{A}\end{array}$ \\
\hline 180 (M5) & $\begin{array}{c}215,1 \pm 35,5 a \\
\mathrm{~A}\end{array}$ & $\begin{array}{c}205,7 \pm \\
\mathrm{A}\end{array}$ & $\begin{array}{c}176,3 \pm \\
A\end{array}$ \\
\hline
\end{tabular}

TABELA 5 - Médias, desvios-padrão e resultados do teste estatístico da comparação da glicemia, em miligramas por decilitro, nos grupos experimentais e momentos de sacrifício.

\section{GLICEMIA}

\section{Períodos de sacrifício (dias)}

\begin{tabular}{c|c|c|c|c|c|c}
\hline Grupos & $\begin{array}{c}\text { Início } \\
\left(\mathbf{M}_{\mathbf{0}}\right)\end{array}$ & $\begin{array}{c}\mathbf{7} \\
\left(\mathbf{M}_{\mathbf{1}}\right)\end{array}$ & $\begin{array}{c}\mathbf{1 4} \\
\left(\mathbf{M}_{\mathbf{2}}\right)\end{array}$ & $\begin{array}{c}\mathbf{2 8} \\
\left(\mathbf{M}_{\mathbf{3}}\right)\end{array}$ & $\begin{array}{c}\mathbf{9 0} \\
\left(\mathbf{M}_{\mathbf{4}}\right)\end{array}$ & $\mathbf{1 8 0}$ \\
\hline & $145,0 \pm 26,3 \mathrm{a}$ & $132,1 \pm 28,5 \mathrm{a}$ & $133,9 \pm 28,8 \mathrm{a}$ & $127,2 \pm 22,9 \mathrm{a}$ & $126,2 \pm 29,8 \mathrm{a}$ & $129,8 \pm 16,4 \mathrm{a}$ \\
$\mathbf{N}$ & $\mathrm{A}$ & $\mathrm{A}$ & $\mathrm{A}$ & $\mathrm{A}$ & $\mathrm{A}$ & $\mathrm{A}$ \\
\hline & $128,3 \pm 24,9 \mathrm{a}$ & $128,7 \pm 19,7 \mathrm{a}$ & $127,9 \pm 17,9 \mathrm{a}$ & $149,9 \pm 62,3 \mathrm{a}$ & $149,7 \pm 95,1 \mathrm{a}$ & $128,8 \pm 28,8 \mathrm{a}$ \\
$\mathbf{A}$ & $\mathrm{A}$ & $\mathrm{A}$ & $\mathrm{A}$ & $\mathrm{A}$ & $\mathrm{A}$ & $\mathrm{A}$ \\
\hline & $142,5 \pm 30,4 \mathrm{a}$ & $131,9 \pm 23,2 \mathrm{a}$ & $124,4 \pm 18,9 \mathrm{a}$ & $159,5 \pm 57,0 \mathrm{a}$ & $132,3 \pm 39,9 \mathrm{a}$ & $135,7 \pm 25,1 \mathrm{a}$ \\
$\mathbf{L}$ & $\mathrm{A}$ & $\mathrm{A}$ & $\mathrm{A}$ & $\mathrm{A}$ & $\mathrm{A}$ & $\mathrm{A}$ \\
\hline
\end{tabular}

TABELA 6 - Médias, desvios-padrão e resultados do teste estatístico da comparação da glicose urinária, em miligramas por decilitro, nos grupos experimentais e momentos de sacrifício. 


\section{GLICOSE URINÁRIA}

\section{Períodos de sacrifício (dias)}

\begin{tabular}{c|c|c|c|c|c|c}
\hline \multirow{2}{*}{ Grupos } & $\begin{array}{c}\text { Início } \\
\left(\mathbf{M}_{\mathbf{0}}\right)\end{array}$ & $\begin{array}{c}\mathbf{7} \\
\left(\mathbf{M}_{\mathbf{1}}\right)\end{array}$ & $\begin{array}{c}\mathbf{1 4} \\
\left(\mathbf{M}_{\mathbf{2}}\right)\end{array}$ & $\begin{array}{c}\mathbf{2 8} \\
\left(\mathbf{M}_{\mathbf{3}}\right)\end{array}$ & $\begin{array}{c}\mathbf{9 0} \\
\left(\mathbf{M}_{\mathbf{4}}\right)\end{array}$ & $\mathbf{1 8 0}$ \\
& $16,7 \pm 6,9 \mathrm{a}$ & $18,6 \pm 14,0 \mathrm{a}$ & $18,9 \pm 12,4 \mathrm{a}$ & $21,8 \pm 14,1 \mathrm{a}$ & $23,4 \pm 9,4 \mathrm{a}$ & $19,4 \pm 7,2 \mathrm{a}$ \\
$\mathbf{N}$ & $\mathrm{A}$ & $\mathrm{A}$ & $\mathrm{A}$ & $\mathrm{A}$ & $\mathrm{A}$ & $\mathrm{A}$ \\
\hline & $18,3 \pm 12,2 \mathrm{a}$ & $19,8 \pm 13,7 \mathrm{a}$ & $25,8 \pm 14,1 \mathrm{a}$ & $24,6 \pm 15,6 \mathrm{a}$ & $22,3 \pm 12,6 \mathrm{a}$ & $23,4 \pm 11,8 \mathrm{a}$ \\
$\mathbf{A}$ & $\mathrm{A}$ & $\mathrm{A}$ & $\mathrm{A}$ & $\mathrm{A}$ & $\mathrm{A}$ & $\mathrm{A}$ \\
\hline & $21,3 \pm 14,4 \mathrm{a}$ & $25,7 \pm 21,3 \mathrm{a}$ & $19,8 \pm 7,8 \mathrm{a}$ & $21,1 \pm 12,3 \mathrm{a}$ & $18,4 \pm 7,1 \mathrm{a}$ & $18,8 \pm 7,0 \mathrm{a}$ \\
$\mathbf{L}$ & $\mathrm{A}$ & $\mathrm{A}$ & $\mathrm{A}$ & $\mathrm{A}$ & $\mathrm{A}$ & $\mathrm{A}$ \\
\hline
\end{tabular}

TABELA 7 - Médias, desvios-padrão e resultados do teste estatístico da comparação da insulina plasmática, em microunidades por mililitro, nos grupos experimentais e momentos de sacrifício.

\section{INSULINA}

\section{Períodos de sacrifício (dias)}

\begin{tabular}{c|c|c|c|c|c|c}
\hline \multirow{2}{*}{ Grupos } & $\begin{array}{c}\text { Início } \\
\left(\mathbf{M}_{\mathbf{0}}\right)\end{array}$ & $\mathbf{7}$ & $\mathbf{1 4}$ & $\mathbf{2 8}$ & $\mathbf{9 0}$ & $\mathbf{1 8 0}$ \\
& $\left(\mathbf{M}_{\mathbf{1}}\right)$ & $\left(\mathbf{M}_{\mathbf{2}}\right.$ & $\mathbf{( \mathbf { M } _ { \mathbf { 3 } } )}$ & $\mathbf{( \mathbf { M } _ { \mathbf { 4 } } )}$ & $\mathbf{( \mathbf { M } _ { 5 } )}$ \\
\hline \multirow{2}{*}{$\mathbf{N}$} & $9,4 \pm 3,7 \mathrm{a}$ & $10,4 \pm 3,1 \mathrm{a}$ & $9,4 \pm 4,7 \mathrm{a}$ & $9,3 \pm 3,4 \mathrm{a}$ & $11,8 \pm 4,5 \mathrm{a}$ & $10,7 \pm 4,1 \mathrm{a}$ \\
& $\mathrm{A}$ & $\mathrm{A}$ & $\mathrm{A}$ & $\mathrm{A}$ & $\mathrm{A}$ & $\mathrm{A}$ \\
\hline & $9,0 \pm 3,4 \mathrm{a}$ & $9,7 \pm 3,0 \mathrm{a}$ & $9,9 \pm 2,8 \mathrm{a}$ & $10,2 \pm 3,3 \mathrm{a}$ & $8,7 \pm 3,7 \mathrm{a}$ & $8,0 \pm 2,6 \mathrm{a}$ \\
$\mathbf{A}$ & $\mathrm{A}$ & $\mathrm{A}$ & $\mathrm{A}$ & $\mathrm{A}$ & $\mathrm{A}$ & $\mathrm{A}$ \\
\hline & $10,7 \pm 4,8 \mathrm{a}$ & $8,8 \pm 2,3 \mathrm{a}$ & $8,6 \pm 2,4 \mathrm{a}$ & $9,3 \pm 3,7 \mathrm{a}$ & $7,2 \pm 1,9 \mathrm{a}$ & $7,3 \pm 2,1 \mathrm{a}$ \\
$\mathrm{L}$ & $\mathrm{A}$ & $\mathrm{A}$ & $\mathrm{A}$ & $\mathrm{A}$ & $\mathrm{A}$ & $\mathrm{A}$ \\
\hline
\end{tabular}

\section{Discussão}

Nosso estudo mostrou que, independentemente da abordagem utilizada, não observamos, a longo prazo, quaisquer alterações clínicas em coelhos com os ductos pancreáticos abertos ou ligados, em relação aos animais normais, apenas manipulados.

Este achado, no entanto, tem sido a regra observada na maioria dos trabalhos experimentais, onde as variáveis estudadas foram avaliadas ${ }^{15,16,9,17,18,19,20,21}$. No homem, 
porém, em razão dos inúmeros fatores envolvidos na sua evolução clínica, tais como alterações ligadas à própria doença de base, ao procedimento cirúrgico de maior parte, à imunossupressão (no caso dos transplantes), às complicações cirúrgicas, à infecção, etc., não é possível estabelecer uma nítida relação de causa-efeito do uso destes métodos, como a estabelecida em nossos achados experimentais. Por estas razões, nos ateremos apenas aos trabalhos experimentais.

Sabidamente, os animais de laboratório são mais resistentes que o homem, frente ao trauma cirúrgico: suportam mais a agressão cirúrgica; recuperam-se mais rapidamente da anestesia; reassumem a atividade e o apetite mais prontamente; são mais resistentes à infecção; e estão menos expostos às agressões externas. Estas peculiaridades, por si só, explicariam por que a curva ponderal de animais operados, em geral, pouco é afetada após os procedimentos cirúrgicos, tendo o retorno precoce à ingesta um dos pontos mais importantes desta performance.

Curiosamente, porém, animais submetidos à ligadura ductal pancreática, não apresentaram alterações compatíveis com insuficiência exócrina do pâncreas, manifestadas por diarréia, má-absorção, distensão abdominal e subnutrição, durante todo o seguimento de 6 meses. Este achado, porém, pode ser explicado pelas diferenças observadas na dieta e no processo digestivo de homens e animais. O balanceamento da dieta é um dos fatores mais importantes. Em condições normais, animais de laboratório recebem rações balanceadas, com teor de nutrientes padronizados, que favorecem a absorção intestinal.

Por outro lado, segundo Fichaux, Bonnafous ${ }^{22}$, 1992, a atividade do sistema biliar de animais exerce importante papel na digestão de gorduras, em proporções até mais acentuadas do que no homem. Este fato sugere que animais poderiam prescindir da atividade pancreática para a absorção deste nutriente, o que é perfeitamente cabível em coelhos onde a ação da bile precede, em muito, a ação das enzimas pancreáticas, já que o sistema biliar desemboca separadamente da via pancreática.

No caso específico dos transplantes pancreáticos, com ductos ligados, as manifestações de insuficiência exócrina obviamente nunca estão presentes, tanto clínica, como experimentalmente, porque nos procedimentos cirúrgicos são conservados os órgãos nativos "in situ”, com a função exócrina preservada.

Este fato ficou bastante documentado nos experimentos conduzidos em porcos, por Kyriakides et $\mathrm{al}^{15,16,17}$, onde os transplantes pancreáticos com ductos ligados eram realizados, tanto em animais pancreatectomizados, como naqueles com os órgãos nativos 
conservados, tendo como controles porcos pancreatectomizados, mas sem transplante. Nesses últimos, eram evidentes as altas taxas de mortalidade por insuficiência pancreática exócrina, caquexia e hiperglicemia, quando comparadas com porcos transplantados sem o pâncreas nativo - onde a insuficiência exócrina também existia, mas era de menor magnitude, e possível de ser controlada com suplementação enzimática exógena - e com aqueles cujos órgãos nativos foram mantidos "in situ", onde as alterações exócrinas não ocorriam.

Brekke et $a^{23,19} 1980,1981$, a exemplo dos nossos achados, também observaram que a supressão da atividade enzimática do pâncreas não interfere com a curva ponderal e com os demais parâmetros clínicos (ingestão hídrica, ingestão alimentar e diurese) de ratos transplantados com o ducto pancreático ligado.

Dubernard et $\mathrm{al}^{24} 1979$, em contraste, observaram importante insuficiência exócrina, com caquexia e perda de peso, em cães normais com o ducto pancreático obliterado por neoprene e depois ligado; indicando, talvez, que a dependência enzimática do pâncreas cresce na escala zoológica, dos animais de pequeno para os de maior porte. Dubernard provou isto nesse mesmo trabalho quando fez suplementação da dieta com extratos pancreáticos e triglicerídios de cadeia média, observando boas condições clínicas dos animais durante todo o experimento.

A despeito de eventuais divergências, o nosso trabalho experimental demonstrou a possibilidade de coelhos normais, submetidos às duas variedades diferentes de tratamento da secreção exócrina do pâncreas, levarem uma vida normal, sem qualquer alteração clínica que os distinguissem de animais normais de igual idade de seguimento.

Numa análise crítica, porém, não esteve ao alcance de nossos recursos técnicos e materiais a realização de provas funcionais do pâncreas, tais como: o teste da secretinapancreozimina, do consumo de aminoácidos, o teste de Lundh e as medidas diretas da lipase sangüínea, isoamilase, tripsina, quimiotripsina e elastase fecal, e as medidas da ação enzimática, através do teste do bentiromide (PABA), do pancreolauril e o teste respiratório. Estes testes, embora fidedignos para a avaliação da função pancreática exócrina, são impraticáveis em nosso meio, inclusive na prática clínica, por serem, a maioria deles, de difícil execução e alto custo.

Laboratorialmente, a amilase e a lipase sangüínea, ao lado do teste do Sudam, com pesquisa de gorduras neutras nas fezes, são os testes "screenings" mais comumente utilizados para avaliar a função pancreática exócrina. Estes testes, porém, somente são 
sensíveis para detectar alterações pancreáticas exócrinas somente quando $80 \%$ ou mais da glândula estiverem afetados e/ou com perdas diárias de gorduras nas fezes superiores a 75g. Além disso, à exceção da amilase, estes testes ainda não estão disponíveis na maioria dos serviços, sendo que no caso do teste do Sudam, o mesmo exigiria, especificamente para o coelho, o uso de gaiolas metabólicas para evitar o contato das fezes com a urina, parâmetro também não dimensionado nesta pesquisa, em razão desta indisponibilidade técnica e material.

Assim, a avaliação funcional do componente exócrino do pâncreas se restringiu, apenas, à dosagem da amilase sangüínea que, ao lado da avaliação clínica, são os exames mais acessíveis para a maioria dos laboratórios.

O comportamento da amilase sangüínea no nosso estudo foi semelhante nos dois grupos tratados deste experimento, sem qualquer variação. Tanto coelhos com o ducto ligado ou deixado livre na cavidade peritoneal apresentaram elevação abrupta dos níveis desta enzima no $1^{\underline{0}}$ e $2^{\underline{0}}$ dia do procedimento cirúrgico, para depois apresentarem redução progressiva dos valores, em níveis compatíveis com a normalidade, a partir do $7^{\stackrel{0}{ }}$ P.O.

Este achado, consequiência imediata da pancreatite aguda que se segue à oclusão aguda do ducto de Wirsung, tem sido descrita, rotineiramente, em homens e animais, por todos os autores interessados neste assunto ${ }^{25,26,9,15,16,17,23,19,24,27}$. Interessante, também, que mesmo animais com o ducto pancreático deixado livre na cavidade peritoneal, apresentaram valores elevados da amilase sangüínea nos primeiros dias de pós-operatório. Este achado também tem sido descrito por inúmeros autores que utilizaram esta técnica ${ }^{28,20,21}$ independentemente de o ducto ter permanecido livre de fibrose ou ter-se comportado como uma técnica de ducto fechado. É provável, contudo, que a absorção peritoneal de enzimas pancreáticas tenha papel relevante na elevação da amilase, enquanto o ducto permanecer aberto. Por outro lado, após a primeira semana do procedimento, com a regressão do processo inflamatório agudo do pâncreas, e a substituição progressiva pelo processo inflamatório crônico e fibrose, os níveis de amilase tendem a se normalizar.

Aumento significativo da amilase sérica foi notado por Brekke et $\mathrm{al}^{19}$ 1980, estudando ratos diabéticos transplantados, com o ducto ligado, tendo observado valores que alcançaram até 15.000 UA/l durante os primeiros dias após o transplante, retornando aos valores normais dentro de 7-8 dias. 
Todavia, como comentamos anteriormente, em relação aos transplantes, não é possível imputar exclusivamente aos procedimentos de abordagem do ducto, o aumento da amilase sangüínea ${ }^{26,27,29,30,31}$.

Criticável, também, do ponto de vista da sofisticação tecnológica, os exames por nós utilizados para aferir a função endócrina do pâncreas ficaram aquém do descrito na literatura, para homens e animais. Em nossa pesquisa analisamos o perfil basal glicêmico no sangue e na urina de todos os animais, durante os 6 meses de seguimento, e também a dosagem dos níveis basais de insulina plasmática por radioimunoensaio.

Desta forma, as conclusões acerca da função endócrina do pâncreas de animais com o ducto ligado, ou deixado livre na cavidade peritoneal, deste trabalho, estão limitadas às condições basais do metabolismo dos carboidratos, haja vista a nossa impossibilidade de realizar dosagens mais sofisticadas, basais e estimuladas, como: a do peptídeo C (precursor da insulina), do glucagon, da saturação glicolítica da hemoglobina (hemoglobina glicada) e os testes de tolerância à glicose (GTT).

Os efeitos das técnicas de supressão da secreção pancreática exócrina sobre a função endócrina do pâncreas, constituem-se um dos aspectos mais importantes da discussão sobre a validade destes métodos, tanto para o tratamento da doença pancreática crônica, como do diabetes mellitus, através do transplante pancreático.

A grande questão, em discussão há vários anos na literatura, é saber se a fibrose e a atrofia do parênquima pancreático, que se seguem à ligadura do ducto de Wirsung, poderiam ou não comprometer a função das ilhotas de Langerhans, a longo prazo. Infelizmente, porém, os resultados das pesquisas são contraditórios e conflitantes e a questão continua em aberto para investigações, como a que efetuamos.

A experiência com a ligadura do ducto pancreático também é relativamente pequena na literatura, sendo os estudos experimentais a sua grande maioria.

Kyriakides et $\mathrm{al}^{15,16,17}$ detêm a maior experiência com este tipo de procedimento. Segundo esses autores, a ligadura ductal pancreática, em porcos, invariavelmente é seguida de inflamação aguda e alterações fibróticas do pâncreas, que acabam por induzir à falência endócrina da glândula.

Kyriakides comprovou isto em estudos em porcos submetidos ao auto e alotransplante pancreático com o ducto ligado ${ }^{15,16}$ onde observou a perda da função dos enxertos pancreáticos, com severa hiperglicemia, em um período médio de 33 dias. 
Essas alterações, no entanto, bram prevenidas em estudo posterior, nas mesmas condições experimentais, pela administração de altas doses de corticóide e pela supressão da atividade exócrina do pâncreas com glucagon ${ }^{17}$.

No presente trabalho experimental, não observamos quaisquer alterações laboratoriais sugestivas de comprometimento endócrino do pâncreas.

Essas conclusões foram corroboradas por estudo de Brekke et aP ${ }^{5} 1980$, realizado em ratos diabéticos induzidos pela estreptozotocina, e submetidos ao transplante pancreático com o ducto ligado. Esses autores observaram que os valores médios da glicose sangüínea foram, inclusive, significativamente mais baixos que os observados em ratos normais, após um período de seguimento de 16 meses. Positivamente, também, não observaram quaisquer alterações no GTT e nas medidas da insulina basal e estimulada que revelassem qualquer redução da capacidade endócrina do pâncreas, a despeito da total atrofia glandular.

Clinicamente, porém, os achados em animais, incluindo os do nosso trabalho, não se reproduzem no homem. Segundo Sutherland et $\mathrm{al}^{35}$ 1986, apenas 13 transplantes pancreáticos foram realizados no mundo com o ducto pancreático ligado. Todavia, esta prática foi abandonada justamente porque associou-se à menor taxa de sobrevida funcional do enxerto, quando comparada a outras modalidades de tratamento do ducto pancreático, tais como: drenagem entérica, drenagem urinária e injeção de polímero sintético.

Ao nosso ver, no entanto, a casuística mundial foi pequena demais, e o seguimento dos pacientes muito curto, também, para trazer conclusões definitivas que condenassem os bons resultados obtidos em animais.

A técnica de ducto pancreático aberto na cavidade peritoneal surgiu em $1978^{32}$ como um método alternativo para solucionar os eventuais problemas causados pela ligadura ductal e pela obliteração com polímero sintético, em relação ao comprometimento da função endócrina do pâncreas.

Sutherland et $\mathrm{al}^{32}$ 1979, acreditavam que a drenagem livre de secreções pancreáticas para peritônio não se seguiria de fibrose periductal e atrofia pancreática, o que tornaria o comprometimento das ilhotas de Langerhans praticamente impossível. Por razões técnicas, e pelas complicações posteriores, advindas com aplicação do método na prática clínica, a expectativa inicial destes pesquisadores foi em parte frustrada.

Curiosamente, porém, a experiência com o ducto pancreático aberto foi primeiro obtida em uma mulher diabética, submetida ao transplante segmentar do pâncreas. Nesta 
paciente, Sutherland et $\mathrm{aP}^{32}$ 1979, observaram o completo restabelecimento do estado normoglicêmico, caracterizado pela normalização das taxas de açúcar no sangue e na urina, e dos níveis de insulina plasmática. Estes dados se mantiveram estáveis durante toda a vida da paciente, que ironicamente morreu 17,5 anos depois, com o enxerto funcionante, após ter caído de um cavalo.

Sutherland concluiu que o método poderia ser usado de rotina em outras pessoas, haja vista que o peritônio era capaz de absorver as secreções enzimáticas inativas produzidas pelo pâncreas, sem determinar alterações peritoneais e no metabolismo dos carboidratos.

A aplicação subseqüente no homem não foi, porém, de todo benéfica ${ }^{18,28}$ em razão de complicações peritoneais em 50\% dos pacientes. Do ponto de vista endócrino, todavia, não foram observadas complicações nos outros 50\%, enquanto os enxertos estiveram funcionantes. Estudos em cães, realizados por estes mesmos pesquisadores, no mesmo ano, continuaram aconselhando a técnica, ainda que com as reservas das complicações peritoneais, curiosamente não observadas em animais.

Em 1984, du Toit et $\mathrm{al}^{20}$ estudando macacos pancreatectomizados, submetidos ao transplante segmentar do pâncreas, mostraram que a técnica de transplante com o ducto pancreático aberto, poderia oferecer consistente normoglicemia para os animais, por períodos médios que variaram de 9,2 a 35,2 dias, porém, observaram que esses animais apresentavam teste de tolerância à glicose (GTT) alterado. Esta alteração, por outro lado, não pôde ser imputada exclusivamente à técnica de abordagem do ducto, porque outras variáveis estavam envolvidas, incluindo o uso de drogas imunossupressoras, que sabidamente alteram o metabolismo dos carboidratos.

Limmer et $\mathrm{al}^{21} 1985$, por sua vez, não encontraram quaisquer alterações no GTT, nos níveis de glicose sangüínea, e nos níveis basais e estimulados de insulina e de peptídeo $\mathrm{C}$ de ratos diabéticos marrons Norway, submetidos ao transplante heterotópico de pâncreas, durante um período de seguimento que variou de 35 a 56 semanas. A importância relevante deste trabalho é que os transplantes foram executados entre animais de mesma linhagem genética (isogênicos), sem a interferência, portanto, de drogas imunossupressoras no metabolismo dos carboidratos.

Os estudos de Limmer foram parcialmente corroborados pelos achados de Cutfield et $\mathrm{al}^{33} 1985$, quando relataram não ter observado qualquer alteração do GTT em cães 
submetidos ao auto-transplante pancreático com o ducto aberto, durante quatro anos de seguimento, a despeito de significativas alterações na secreção de insulina.

Calhoun et $\mathrm{a}^{34}$ no ano seguinte (1986) usando um modelo similar encontrou, porém, significativa alteração do GTT e produção subnormal de insulina durante quase um ano de pós-operatório.

Assim, em que pese os resultados favoráveis, as contradições e o número limitado de experiências clínicas - quase todas elas restritas ao grupo de Minneapolis, USA ${ }^{35}$ - não há como afirmar definitivamente se a técnica aberta pode ou não se correlacionar com dano endócrino do pâncreas. À luz dos nossos conhecimentos, porém, este fato não ocorre, haja vista a manutenção dos níveis basais da glicose sangüínea e urinária, bem como dos níveis de insulina plasmática, durante todo o seguimento dos nossos animais.

\section{Conclusões}

Baseados nos resultados obtidos e nas condições experimentais do presente trabalho, podemos concluir que durante 6 meses de seguimento: 1) a ligadura ductal e a livre drenagem de secreções para o peritônio não alteraram parâmetros clínicos de coelhos, incluindo: atividade, apetite, evolução da curva ponderal e medidas da ingestão hídrica e alimentar; 2) à exceção da amilase sangüínea, cujos níveis basais foram significativamente elevados no $1^{\underline{0}}$ e $2^{\underline{0}}$ dias de pós-operatório, as duas modalidades técnicas de abordagem da secreção exócrina do pâncreas, utilizadas em coelhos, não determinaram quaisquer alterações dos níveis basais de glicose sanguiínea, glicose urinária e de insulina plasmática.

\section{Agradecimentos}

Os autores agradecem as Sras. Maria Clara Ferreira Chaguri, Irene Spago, Maria Helena Spadot e a bióloga Sônia Maria Capeletti pela assistência técnica no Laboratório Experimental.

\section{Referências}

1. Gall FP, Muhe E, Gebhardt C. Results of partial and total pancreatectomy in 117 patients with chronic pancreatitis. World J Surg 1981; 5: 269-75.

2. Andrén-S Andberg A, Ihse I. Factors influencing survival after total pancreatectomy in patients with pancreatic cancer. Ann Surg 1983;198: 605-10. 
3. Amman RW, Akovbiantz A, Largiadèr F, Schueller G. Course and outcome of chronic pancreatitis. Longitudinal study of a mixed medical-surgical series of 245 patients. Gastroenterology 1984; 86: 820-8.

4. Howard JM. Surgical treatment of chronic pancreatitis. In: Howard JM, Jordan JL, Rebers HH (Eds). Surgical disease of pancreas. Philadelphia, Lea \& Febiger 1987; 496-520.

5. Howard JM. Pancreatojejunostomy: leakage is a preventable complication of the Whipple resection. J Am Col Surg 1997;184: 454-7.

6. Warren KW. Surgical management of chronic relapsing pancreatitis. Am J Surg 1969; 117: 24-32.

7. Rohner A, Spiliopoulos A. Pancrêatite chronique primitive. Schweiz Med Wochenschr 1970; 100: 1203-5.

8. Hollender LF. Chronische Pankreatitis. Dtsch. Med. Wochenschr. 1977; 102: 546-7.

9. Gebhardt C, Stolte M. Pankreasgang-Okklusion durch Injektion einer schnellartenden Aminosäurelösung. Langenbecks Arc Chir 1978; 346:149-66.

10. Petronio R, Vantini J, Marzolli GP, Ptubello W, Pederzoli P, Puchetti V, Nicoli N, Sério G. II trattamento chirurgico delle pancreatiti croniche. Minerol Med 1977; 68: 2109-21.

11. Gebhardt C, Gall Fp, Zirngibl H. Chirurgische Behandlung der chronischen Pankreatitis. Dtsch Ärzteblatt 1983; 8: 21-27.

12. Gebhardt C. Chirurgische Therapie der chronischen Pankreatitis. In: Gebhardt C. Die Chirurgie des Exokrinen Pankreas G, Thieme, Stuttgart - New York 1984; 139-91.

13. Brooks JR Presidential address: Where are we with pancreas transplantation? Surgery 1989; 106:935-945.

14. Sutherland DER, Gruessner RWG, Dunn DL, Matas J, Abdnav H, Kandaswamy R, Mauer S, Kennedy WR, Goetz FC, Robertson P, Gruessner AC, Najarian JS. Lessons learned from more than 1,000 pancreas transplants at a single institution. Ann Surg 2001; 233: 463-501.

15. Kyriakides GK, Arora VK, Lifton J, Nuttal FQ, Miller J. Porcine pancreatic transplantation. I Allotransplantation of duct ligated segments. J Surg Res 1976a; 20: 451-60. 
16. Kyryakides GK, Arora VK, Lifton J, Nuttal FQ, Miller J. Porcine pancreatic transplantation. II Autotransplantation of duct ligated segments. J Surg Res 1976b; 20: 461-72.

17. Kyriakides GK, Nutrall FQ, Miller J. Segmental pancreatic transplantation in pigs. Surgery 1979;85:(2)154-9.

18. Sutherland DER, Baumgartner D, Najarian JS. Free intraperitoneal drainage of segmental pancreas grafts: clinical and experimental observations on technical aspects.Transplant Proc 1980; 12: 26-32.

19. Brekke IB, Oyasoeter S. Effect of pancreas transplantation on plasma lipids and plasma concentrations of pancreatic hormones in streptozotocin diabetic rats. Europ Surg Res1981;13:361-70.

20. du Toit DF, Heudenrych JJ, Ben Smit Louw G, Zuurmond T, Laker L, Els D, Weideman A, Wolfe-Coote S, van der Merwe E, Groenewald WA. Experimental vascularized segmental pancreatic and islet transplantation in the baboon. World $\mathbf{J}$ Surg 1984; 8: 236-43.

21. Limmer J, Beischer W, Büchler M, Beger HG. Heterotopic pancreas transplantation in the rat. Transplantation Proceedings 1985; 17: 400-1.

22. Fichaux F, Bonnafous R. Responsiveness and memory of the pancreatic B-cells to the insulinsecretagogues D-glucose and L-arginine in prediabetic on diabetic rabbits. Pancreas 1992; 7: 585-94.

23. Brekke IB, Gullesen I, Refsum SB, Flatmark A. Long-term endocrine function of duct-ligated pancreas isotransplants in rats. Europ Surg Res 1980; 12: 167-78.

24. Dubernard JM Traeger J, Neyra P, Touraine JL, Blanc N, Devonec M. Long-term effect of neoprene injection in the canine pancreatic duct. Transplantation Proceedings 1979; 11: 1498-9.

25. Kelly WD, Lillehei RC, Merkel FK, Idezuki Y, Goetz FC. Allotransplantation of the pancreas and duodenum along with the kidney in diabetic nephropathy. Surgery 1967; 61: 827-37.

26. Groth CG, Lundgren G, Arner P, Collste H, Hardstedt C, Lewander R, Östman J. Rejection of isolated pancreatic allografts in patients with diabetes. Surg Ginecol Obstet 1976; 143: 933-40.

27. Dubernard JM, Traeger J, Piatti PM, Gelet A, El Yafi S, Martin X, Devonec M, Henriet M, Kamel G, Canton F, Codas H, Touraine JL. Report of 54 human segmental 
pancreatic allografts prepared by duct obstruction with neoprene. Transplantation Proc $1985 ; 17: 312-4$.

28. Sutherland DER, Goetz FC, Rynasiewicz JJ, Baumgartner D, Elick BA, White DC, Najarian JS. Segmental pancreas transplantation from living related and cadaver donors: a clinical experience. Surgery 1981; 90: 159-69.

29. Illner WD, Schleibner S, Abendronth D, Landgraf R, Land W. Recent improvement in clinical pancreas transplantation. Transplant Proc 1987; 19:3870-1.

30. Tydén G, Brattström C, Bolinder J, Bohman SO, Groth CG, Brekke IG, Holdaas H, Flatmark A. Long-term metabolic control in recipients of segmental-pancreas grafts with pancreaticoenterostomy or duct obstruction. Diabetes 1989; 36(suppl. 1): 94-6.

31. Secchi A, Dubernard JM, La Rocca E, Lefrancois N, Melandri M, Martin X, Touraine JL, Traeger J, Pozza G. Endocrinometabolic effects of whole versus segmental pancreas allotransplantation in diabetic patients - A two-year follow-up. Transplantation 1991; 51(3): 625-9.

32. Sutherland DER, Goetz FC, Najarian JS. Intraperitoneal transplantation of immediately vascularized segmental pancreatic grafts without duct ligation: a clinical trial. Transplantation 1979; 28: 485-91.

33. Cutfield RG, Polonsky K, Olson L, Kyriakides G, Miller J, Mintz DH. Long-term follow-up of canine segmental pancreatic autographs. Diabetes 1985; 34:174-8.

34. Calhoun P, Brown KS, Krush DA, Barido E, Farris H, Schenk WG 3rd, Rudolf LE, Andersen DK, Hanks JB. Evaluation of insulin secretion after pancreas autotransplantation by oral or intravenous glucose challenge. Ann Surg 1986; 204 : 585-93.

35. Sutherland DER, Goetz FC, Najarian JS. Pancreas transplantation. Clin Chem 1986; 32(10B): B83-B96. 49.

Correspondência:

Lauro Bogodar Kuczynski

Dr. Raul Carneiro Filho, 328

80620-440 Curitiba - PR

Tel: (41)333-3322

drkuczynskilb@uol.com.br

Data do recebimento: 05/02/2004

Data da revisão: 22/02/2004

Data da aprovação: 18/03/2004 
Conflito de interesse: nenhum

Fonte de financiamento: CAPES

\section{Como citar este artigo:}

Kuczynski LB, Spadella CT, Padovanni CR. Efeitos da ligadura do ducto pancreático e da secção ductal com livre drenagem de secreções para o peritônio sobre as funções endócrina e exócrina do pâncreas: estudo clínico e laboratorial em coelhos. Acta Cir Bras [serial online] 2004 Maio-Jun;19(3). Disponível em URL: http://www.scielo.br/acb [também em CD-ROM]. 